\title{
Scientific Progress: Four Accounts
}

\author{
Forthcoming in Philosophy Compass
}

Finnur Dellsén

\begin{abstract}
Scientists are constantly making observations, carrying out experiments, and analyzing empirical data. Meanwhile, scientific theories are routinely being adopted, revised, discarded, and replaced. But when are such changes to the content of science improvements on what came before? This is the question of scientific progress. One answer is that progress occurs when scientific theories 'get closer to the truth', i.e. increase their degree of truthlikeness. A second answer is that progress consists in increasing theories' effectiveness for solving scientific problems. A third answer is that progress occurs when the stock of scientific knowledge accumulates. A fourth and final answer is that scientific progress consists in increasing scientific understanding, i.e. the capacity to correctly explain and reliably predict relevant phenomena. This paper compares and contrasts these four accounts of scientific progress, considers some of the most prominent arguments for and against each account, and briefly explores connections to different forms of scientific realism.
\end{abstract}

Keywords: scientific progress; truthlikeness; problem-solving; knowledge; understanding; theory change; scientific realism.

\section{Introduction}

John Dalton is proposed the first modern atomic theory of matter in the beginning of the $18^{\text {th }}$ century. 'Atom' literally means indivisible, and Dalton's theory was that atoms were the very smallest constituents of matter. However, J.J. Thomson's experiments with cathode rays around a century later led him to conclude that atoms contained even smaller particles with a negative charge - electrons - and that these were evenly distributed within a positively charged area or substrate. According to Thomson, Dalton's original atomic theory was strictly speaking wrong in taking atoms to be indivisible. A few years later, Thomson's theory would again be revised by Ernest Rutherford, whose experiments with shooting alpha particles through a gold foil suggested that most of the atom's mass must be concentrated in a positively-charged core - the nucleus. So, just as Thomson revised Dalton's ideas about the atom, so too did Rutherford revise Thomson's. This process continued with Niels Bohr's revisions of Rutherford's theory in his quasi-quantum theory of the atom, and with Erwin Schrodinger's proposal of a fully quantum mechanical theory in 1926. 
Suppose you think, as most of us presumably do think, that each step in this process was an improvement over the previous step - that it would be a mistake to reverse the process and go back to, say, Thomson's 'plum pudding model' (so called because the electrons were taken to behave as if they floated in a fluid, much like the plums in a plum pudding). If so, then you think that these episodes involve more than mere changes in our scientific theories - you think that they involve scientific progress. Or, to be more precise, you think the episodes involve cognitive scientific progress - the kind of scientific progress that has to do with improvement in our theories, hypotheses, or other representations of the world, ${ }^{1}$ rather than other improvements of or within science (such as increased funding for scientific research, better educational structures for scientists, or increased gender equality among scientific researchers). There are many ways for science to improve, but our interest here is specifically with cognitive improvements in science.

But when exactly is one scientific theory or representation a (cognitive) improvement over another? What is scientific progress? Initially, this question might seem to admit of an obvious answer: Science makes cognitive progress when scientists discover the truth about something. Call this the naïve view of scientific progress. There is surely something right about the naïve view, but notice that it it's not clear how it would explain how Dalton's, Thomson's, Rutherford's, and Bohr's theories all contributed to scientific progress. After all, every one of these theories is strictly speaking false (and the same may yet turn out to be true of Schrodinger's), so we can't say that each step constituted the kind incremental scientific progress that we were initially inclined to attribute to them. This problem with the naïve view motivates the idea that perhaps scientific progress amounts to getting closer to the truth each step is progressive because it brings us a better approximation to the truth than we had before. That idea is at the heart of the truthlikeness account of scientific progress, proposed by Karl Popper and developed primarily by Ilkka Niiniluoto (see section 2).

A different approach to scientific progress emphasizes the possibility of radical change in the theoretical frameworks in which different theories are proposed and evaluated. In contrast to episodes in which later theorists proposed relatively modest revisions of earlier theories, science sometimes experiences changes to the basic metaphysical, conceptual, and methodological foundations of a given discipline. Thomas Kuhn famously referred to such changes as 'scientific revolutions' and insisted that we cannot, even in principle, compare the truth content of scientific theories before and after a revolution. For this reason, Kuhn suggested that a viable account of scientific progress would define it not in terms of truth or truthlikeness - understood as requiring some form of (partial) correspondence between as mind-independent world and our representations of it - but rather in terms of later theories being more effective for solving the scientific puzzles that arise within a given framework. Kuhn's idea is fleshed out by Larry Laudan in his problem-solving account of scientific progress (see section 3).

In the recent literature on scientific progress, two new views have emerged. Alexander Bird has defended an epistemic account according to which science makes progress precisely

\footnotetext{
${ }^{1}$ To simplify the discussion in what follows, I will often use the term 'theory' in a very liberal sense to include any scientific claim, including e.g. observation reports.
} 
when knowledge is accumulated. In some ways, this represents a return to the naïve view, since Bird's view requires that each progressive step adds a fully true proposition to science's knowledge corpus. However, the epistemic account is also more demanding than the naïve view, since knowledge is taken to amount to more than truth (see section 4). The possibility has also been raised of defining scientific progress in terms of understanding rather than knowledge - a thought that undergirds the noetic account of scientific progress. Here the idea is that science makes progress precisely when we gain more understanding, which roughly amounts to increasing the explanatory and predictive capacities of our theories (see section 5).

In what follows, I outline these four different accounts of scientific progress and highlight some important ways in which they differ. Each account places its own distinctive type of cognitive achievement at the heart of scientific progress - truthlikeness, problemsolving, knowledge, or understanding. In order to draw out the most significant differences between these accounts, we will present each account as an attempt to subsume all cases of scientific progress under just one type of cognitive achievement (e.g. knowledge in the case of the epistemic account). Of course, the most plausible account of scientific progress may well be 'pluralistic' in the sense of taking more than one type of cognitive achievement as constituting progress. For example, one could easily imagine a modified version of the truthlikeness account according to which the central and most significant cases of scientific progress are increases in truthlikeness, but other cognitive achievements such as epistemic justification can also constitute a kind of scientific progress (albeit perhaps of a lesser kind or degree). For lack of space, we will not be examining hybrid accounts of this sort here.

\section{Progress and Truthlikeness}

Even when two theories are both false, there is an intuitive sense in which one of them may be 'closer to the truth' than the other. The truthlikeness account of scientific progress is based on the idea that progress is made when our scientific theories become more truthlike (or verisimilar) in this intuitive sense. For example, if a later theory includes additional correct content that a previous theory did not contain, and the later theory includes no additional false content, then the later theory may be said to be more truthlike than then previous theory. Such a change would thus be progressive on the truthlikeness account. Similarly if the later theory includes all the correct content of the previous theory and excludes some of the incorrect content. Popper (1972) proposed a definition of truthlikeness that was intended to deliver on these intuitions, but it was later shown that Popper's definition could not make any distinctions in truthlikeness among theories that were strictly speaking false (Tichý 1974; Miller 1974). Since those are often precisely the sort of comparisons that are needed to analyze scientific progress, it became clear that Popper's definition of truthlikeness could not be used to flesh out the idea that scientific progress consists in increasing truthlikeness.

In response to this problem, a new approach to truthlikeness was developed by Niiniluoto $(1977 ; 1987)$ and Oddie $(1986)$ based on the idea that the states of affairs described a more truthlike theory would be more similar to the actual state of the world than that 
described by a less truthlike theory. Somewhat more precisely, we start with a language $L$ in which the theories in question can be formulated as disjunctions of descriptions of possibilities $C_{i}$ about what the world is like in all its details. In this language $L$, there is one and only one possibility, $C^{*}$, that actually describes the whole truth about the world in all its details. The degree of truthlikeness of a theory is then defined in terms of how well the possibilities associated with each theory match the complete truth $C^{*}$, either on average (Oddie 1986) or in a more complex way (Niiniluoto 1987). We end up with a function $\operatorname{Tr}\left(T, C^{*}\right)$ whose output is a number between 0 and 1 that is meant to measure the truthlikeness of $T$ relative to the complete truth $\mathrm{C}^{*}$. With this technical machinery, the thought that science progresses when we get closer to the truth can be stated as follows (see esp. Niiniluoto 1980, 1984, 2015):

The truthlikeness account: Science makes (cognitive) progress precisely when a theory

$\mathrm{T}$ is replaced with a more truthlike theory $\mathrm{T}^{\prime}$, i.e. $\operatorname{Tr}\left(\mathrm{T}, \mathrm{C}^{*}\right)<\operatorname{Tr}\left(\mathrm{T}^{\prime}, \mathrm{C}^{*}\right)$.

This account is also known as the verisimilitudinarian approach to scientific progress.

Note that the truthlikeness account is meant to specify when progress in fact takes place; not whether we have reason to think that an episode is progressive. Thus there may be scientific progress from $T$ and $T^{\prime}$ even if we have reasons to think the step is non-progressive, or even regressive. However, the above definition of truthlikeness also makes it possible to define what it is for a change from one theory to another should seem progressive given our evidence. To that end, a notion of expected truthlikeness is defined as the probabilityweighted sum of the truthlikeness of a given theory $T$ given different possibilities $C_{i}$ about what the world is like in all its details:

$\operatorname{ExTr}(T, E)=\sum i \operatorname{Pr}\left(C_{i} \mid E\right) \operatorname{Tr}\left(T, C_{i}\right)$

where the probabilities $\operatorname{Pr}\left(C_{i} \mid E\right)$ are meant to measure how likely the scientists should find each possibility $C_{i}$ given their evidence $E$. We can then say that, on the truthlikeness account, replacing $T$ with $T^{\prime}$ should seem progressive, given some evidence $E$, just in case $\operatorname{Ex} \operatorname{Tr}(T, E)<$ $\operatorname{ExTr}\left(T^{\prime}, E\right)$.

As this illustrates, the truthlikeness account can be fleshed out in an impressively precise way. However, there are also serious doubts about whether the notion of truthlikeness is too language dependent to account for scientific progress. In particular, Miller (e.g. 2006: 197-235) has vigorously pressed the objection that the degree of truthlikeness of a given theory $T$, and its comparative truthlikeness with another theory $T^{\prime}$, is sensitive to the language $L$ in which $T$ and $T^{\prime}$ are being compared to the complete truth $C^{*}$. Thus a step from $T$ to $T^{\prime}$ may be progressive when the theories are formulated in one language, whereas in another language the very same step would be regressive (it decreases truthlikeness). One possible solution to the problem would be to claim that there is a unique language - call it $L^{*}$ - relative to which objective judgments of truthlikeness should all be made. However, it is at least questionable whether there is any such privileged language in science; moreover, since we presumably cannot tell what language enjoys this exalted status, evaluating truthlikeness relative to such a language would at best seem to be exceedingly difficult. 


\section{Progress and Problem-Solving}

A different approach to scientific progress focuses not on truth or truthlikeness, but on whether theories enable scientists to solve various problems. The basic idea is that a change from one theory to another is progressive just in case the later theory solves more problems than the earlier theory. For example, if we ask why there was progress when Rutherford's theory of the atom was replaced with Bohr's, the problem-solving account would consider the problems that Bohr's theory could solve but Rutherford's couldn't. Importantly, Bohr's theory solves the problem of why hydrogen and several other chemical elements emit radiation with certain fixed wavelengths. In Bohr's theory, electrons have quantized angular momentum, which means that an electron can only gain or lose energy in fixed discrete quantities corresponding to different radiation wavelengths. This solution to the problem was not available in Rutherford's theory, since the latter does not specify that the angular momentum of the electron is quantized.

While Popper (1972: 260-265) also emphasized the importance of solving problems, the problem-solving account proposes to define scientific progress entirely in terms of scientists' ability to solve such problems from their own point of view. This idea has been most fully developed by Larry Laudan $(1977,1981 b)$, but it is inspired by Thomas Kuhn's notion of 'puzzle-solving'. For Kuhn and Laudan, scientific research is typically carried out against a backdrop of metaphysical, conceptual, and methodological assumptions - 'paradigms' or 'disciplinary matrices' for Kuhn, and 'research traditions' for Laudan - which determine what counts as genuine problem, how important it is that one's theories solve each problem, and indeed what would count as a solution to a given problem. For Laudan, a theory's problemsolving effectiveness is determined by the number and importance of problems solved by the theory, as assessed from within such a research tradition, and by the number and importance of problems that it leaves unsolved. Laudan's account, then, is that progress takes place precisely when our theories' problem-solving effectiveness increases, as assessed from the standpoint of the relevant research tradition.

In fleshing out this idea, Laudan distinguishes between two importantly different kinds of problems that a new theory should seek to solve. Empirical problems are questions concerning the objects or entities that a particular scientific theory is meant to explain or account for, e.g. why it is that some particular chemical elements emit radiation with fixed wavelengths. By contrast, conceptual problems are issues with features of the theories themselves or how they relate to other theories or assumptions within the research tradition. A conceptual problem could either be an internal inconsistency or incongruence within a given theory, or an external incongruence between the theory and some other element of one's research tradition, e.g. another theory or a methodological dictum of the research tradition. Laudan stresses that a new theory's solution to an important conceptual problem can be just as progressive as a solution to an empirical problem.

One apparent benefit of defining scientific progress in terms of problem-solving rather than truth or truthlikeness concerns episodes in which a scientific discipline experiences a change in the research tradition itself. Here we make contact with Kuhn's influential (but controversial) claim that theories before and after a change of scientific paradigm cannot be 
objectively compared in terms of their truth or truthlikeness (Kuhn 1970: 198-207). Whatever we think of this argument in general, it does have some bite against the truthlikeness account as it has been developed so far. After all, it is widely acknowledged that scientific revolutions of the sort that Kuhn was interested in are often accompanied by changes in the conceptual framework in which theories are formulated. As we have seen, Niinluoto's definition of truthlikeness is language-dependent, so one theory could have a greater truthlikeness than another relative to the conceptual framework used by an earlier research tradition but not relative to the framework used by a later tradition. In cases of that sort, it seems that the truthlikeness account would imply that there is no objective answer as to whether the episode really is progressive.

By contrast, while each theory's problem-solving effectiveness is in a certain sense relative to the research tradition in which it is embedded, it is possible to objectively compare the problem-solving effectiveness of the theories-cum-research-traditions in a given domain at different points in time. Some combinations of theories and research traditions will simply leave fewer (and/or less important) problems unsolved, and so the problem-solving account holds that progress occurs when we move toward those combinations. Interestingly, since problem-solving effectiveness is defined in terms of what the research tradition itself determines to be (important) problems and solutions, a progressive episode need not involve any change in the scientific theories accepted before and after the episode. Rather, progress may merely consist of an appropriate change in that part of the research tradition which determines what counts as (important) problems and solutions, as when an unsolved problem is deemed to be a pseudo-problem, or when something that was previously considered an inadequate solution to a problem is deemed to be satisfactory in the later research tradition.

A distinguishing and controversial feature of the problem-solving account is that it eschews any standard truth or accuracy requirement on progressive theories. ${ }^{2}$ That is, later theories need not represent the relevant parts of the world more faithfully than earlier theories in order for progress to take place. Indeed, the problem-solving account even allows for our representations to become less accurate through a scientifically progressive episode. Similarly, the empirical problems that theories are meant to solve need not concern any actual state of affairs; it suffices that they be believed to do so by those who adhere to the research tradition. So according to the problem-solving account, it may count as progressive to adopt or accept an entirely false theory in order to solve a 'problem' with no basis in reality at all. This objection is pressed most forcefully by Bird (2007: 68-70), who illustrates with the case of Nicole d'Oresme's belief that hot goat's blood could split diamonds. Bird's point is that Oresme's adopting a theory that presumes to solve this 'problem' would not constitute scientific progress, regardless of what Oresme himself might have thought.

Interestingly, however, Laudan considers the lack of any such truth or accuracy requirement on scientific progress to be a desirable feature of his account, on the grounds

\footnotetext{
${ }^{2}$ More precisely, proponents of problem-solving accounts reject truth requirements on progress when 'truth' is understood as requiring a correspondence between theories (or other scientific representations) and some part of a mind-independent reality. By contrast, proponents of problem-solving accounts could conceivably accept some kind of truth requirement given a pragmatic or even deflationary theory of truth, for example (see Glanzberg 2016).
} 
that truth "is intrinsically transcendant and hence closed to epistemic access" (Laudan 1981b: 145). ${ }^{3}$ Here and elsewhere, Laudan suggests that the problem with requiring progressive theories to be true or more truthlike is that this would make it impossible to evaluate whether a change is progressive. Put differently, Laudan's idea seems to be that what is in fact progressive should be identified with what could be evaluated as progressive. ${ }^{4}$ In Laudan's view, the history of science contains so many false theories that were once thought to be true that we should be skeptical of any evaluation of our current theories as true or more truthlike than earlier theories. ${ }^{5}$ Thus, argues Laudan, in order to preserve a connection between what is scientifically progressive and what is evaluable as such, we must eschew any truth or accuracy requirement on scientific progress.

\section{Progress and Knowledge}

In 2007, Bird breathed new life into the debate about scientific progress with the publication of the first systematic defense of a knowledge-based account, the epistemic account. ${ }^{6}$ On Bird's view, science progresses precisely when it shows the accumulation of knowledge. The term 'knowledge' is understood in the epistemologists' sense in which knowledge entails truth, justification, and belief. That is, in order to know something, one must believe it, this belief must be true, and the belief must be justified by scientific evidence or arguments (e.g. observations, experiments, or calculations). Most philosophers interested in the nature of knowledge think that knowledge cannot be defined as justified true belief, due to 'Gettier cases' in which beliefs that are true and justified are intuitively not known. Nevertheless, nearly all epistemologists think that justification, truth, and belief are necessary conditions for knowledge - and this view is shared by Bird.

Thus, for Bird, a change from one theory to another only counts as progressive if the later theory contains more or stronger propositions that are true, believed, and justified by the scientific evidence than does the earlier theory. So, for example, if the later theory merely contains more truths than the earlier theory, but these additional truths are either not believed or justified by scientific evidence to the extent required for knowledge, then no progress has taken place on the epistemic account. Bird sees this as an advantage of his account. Indeed, Bird argues against the truthlikeness account by invoking hypothetical cases in which scientists add a new true theory to their theoretical corpus (and so truthlikeness increases), but the new theory is not adequately supported by scientific evidence. Bird claims that our intuitive judgment in such cases is that there would be no progress, and there is some indication that this intuition is widely, albeit not universally, shared (Mizrahi and Buckwalter 2014). To illustrate with Bird's main example, the French scientist Réné Blondlot believed in

\footnotetext{
${ }^{3}$ Elsewhere, Laudan claims that the greatest virtue of his account is that it puts is "in a position to decide whether science is ... progressive" (Laudan 1977: 127).

${ }^{4}$ This identification is explicitly rejected by other contributors to the debate. Indeed, as we have already seen, the truthlikeness account provides separate criteria for each in terms of truthlikeness and expected truthlikeness, respectively.

${ }^{5}$ This is the argument that Laudan developed into his 'pessimistic meta-induction' (Laudan 1981a).

${ }^{6}$ Barnes (1991) and Cohen (1980) had previously suggested that scientific progress should be defined in terms of knowledge in response to issues with the truthlikeness account.
} 
the existence of what he called ' $\mathrm{N}$-rays' on the basis of subjective observations which were arguably known to be highly unreliable in the circumstances. Blondlot's belief was thus plausibly not justified by scientific evidence, even by his own lights. But let's nevertheless suppose that Blondlot's belief was true - that N-rays do in fact exist. In Bird's view, it would still be intuitively wrong to attribute scientific progress to Blondlot's 'discovery'.

Bird's argument raises the issue of whether intuitive judgments should have much probative value in the debate, given that the aim could hardly be to analyze the everyday concept of 'scientific progress', but rather to settle a normative issue about which theoretical changes count as improvements. Moreover, there are a number of ways of resisting Bird's argument that accommodate or explain away the alleged intuition that progressive theories must be supported by adequate evidence. For example, Rowbottom $(2008,2010)$ points out that justification can serve as a means to true or truthlike theories, which in turn would constitute progress on the truthlikeness account. ${ }^{7}$ Similarly, Cevolani and Tombolo 2013 (see also Niiniluoto 2015) argue that the intuition that scientific progress requires justification can be accommodated within the truthlikeness account in virtue of the fact that justification is required for a rational estimation of expected truthlikeness. Yet another response is provided by Dellsén (2016), who argues that we mistakenly hesitate to attribute progress in the absence of justification because we tend to disapprove of the means by which scientists make progress in cases of that sort.

One potential problem with the epistemic account concerns how to handle progressive episodes in which a false theory is replaced with another false theory. In the truthlikeness account, the episode would be progressive if the later theory is more truthlike; in the problemsolving account, it would be progressive if the later theory solved more problems and/or left fewer problems unsolved. The difficulty for the epistemic account is that since knowledge entails truth, no false theory can count as knowledge - regardless of how truthlike it is. Bird's (2007) solution is to identify, in each false theory $T$, a true proposition $T^{-}$that is entailed by $T$ but not vice versa. Given that this more modest proposition $\mathrm{T}^{-}$is strictly true (and not merely truthlike to some degree), it would be possible to know $\mathrm{T}^{-}$- assuming, of course, that other requirements for knowledge are satisfied. Bird's idea, then, is that replacing a false theory with another false one constitutes progress when the known entailments of the theories increase during the change - i.e. when there is accumulation of knowledge at the level of the true entailments of our theories. ${ }^{8}$

\section{Progress and Understanding}

The most recently defended account of scientific progress associates it with understanding. Unfortunately, the concept of 'understanding' is itself a hotly debated topic, so there is little agreement on what is required for someone to possess understanding. However, it is reasonably uncontroversial that understanding is a matter of degree (i.e., that one can have greater and lesser understanding of the same phenomenon); that understanding is either

\footnotetext{
${ }^{7}$ Bird (2008) responds to Rowbottom (2010).

${ }^{8}$ Niiniluoto (2015: 76-77) and Saatsi (2016: 11-12) present some difficulties with this route.
} 
factive or quasi-factive (i.e., that one cannot understand on the basis a false or radically incorrect theory); and that understanding bears some close connection to explanation, even if it may be possible to understand something without having an explanation. Bird (2007) takes understanding to be species of knowledge - knowledge of causes - and argues that the epistemic account can thus accommodate the idea that increasing understanding provides an especially powerful way in which science can progress (see also Bangu 2015). Although Bird's view about the connection between understanding and knowledge is shared by some who have written on understanding specifically (e.g., Grimm 2006; Sliwa 2015), there are several arguments for disassociating knowledge and understanding (e.g., Kvanvig 2009; Hills 2016; Wilkenfeld 2017; Dellsén 2017).

Given the lack of consensus as to the nature of understanding, the most fruitful approach to developing an understanding-based account of scientific progress may be to use 'understanding' in a stipulated sense. This is the route taken by Dellsén (2016), who stipulates that an agent partly understands something just in case she grasps how to correctly explain and/or predict some aspects of the target phenomenon in the right circumstances. On this basis, Dellsén proposes an understanding-based account of scientific progress, the noetic account, according to which an episode in science is progressive just in case scientists grasp how to correctly explain or reliably predict more aspects of the world than they did before. ${ }^{9}$ Unlike Bird's epistemic account, this does not require that scientists have justification for, or even belief in, the explanations or predictions they propose.

Dellsén (2016: 76-77) argues that this difference between his noetic and Bird's epistemic accounts speaks in favor of the former, since there are cases in which scientific progress consists in an improved ability to use an unjustified (but correct) theory to explain or predict phenomena. For example, Einstein's explanation of Brownian motion in 1905 was based on the kinetic theory of heat, which was highly speculative at the time (and thus unjustified), but Dellsén claims that Einstein's achievement nevertheless constituted scientific progress. Dellsén (2016: 77-78) also argues that the noetic account gives the more plausible verdicts in cases where knowledge is accumulated without any corresponding increase in scientific understanding. For example, scientists could come to know about entirely spurious statistical correlations, such as the fact that increases in childbirth rates outside of Berlin city hospitals are correlated with increases in stork populations around the city. Given that this piece of statistical information would constitute knowledge but not provide any understanding, the noetic and epistemic accounts deliver different verdicts about whether it makes for scientific progress. ${ }^{10}$

How is the noetic account related to the truthlikeness and problem-solving accounts of scientific progress? The key thought behind the truthlikeness account is that progress can be made by getting 'closer to the truth'. Since understanding is at least quasi-factive and a matter of degree, this thought can be accommodated relatively easily within the noetic

\footnotetext{
${ }_{9}^{9}$ Potochnik (2015) appeals to the widespread use of idealizations in science to argue that science aims at understanding rather than truth. Given an intuitive connection between scientific progress and the aim of science (see section 6), Potochnik's view may entail something like the noetic account of progress.

${ }^{10}$ Park (2017) objects to these arguments; Dellsén (2018) replies in turn.
} 
account. One's degree of understanding would simply be determined, at least in part, by how close to a fully correct representation of something is used for explanatory and predictive purposes. Indeed, an understanding-based account could simply appeal to the account of truthlikeness developed by proponents of the truthlikeness account, in which case the differences between the noetic account and the truthlikeness account would be fairly modest. The most important difference between the two accounts would concern whether proposing new explanations or making new predictions could itself constitute progress, even when there is no change in the theories with which one would explain and predict. ${ }^{11}$ In this respect, the noetic account would resemble the problem-solving account, which similarly defines scientific progress in terms of what scientific theories are used for. Indeed, Laudan's notion of solving an empirical problem resembles, and sometimes reduces to, potentially explaining an empirical phenomenon (Laudan 1977: 14-26).

\section{Connections and Unexplored Territory}

The debate about scientific progress is intimately related to other debates in philosophy of science. For one thing, there seems to be a close conceptual connection between scientific progress and the aim of science. Roughly, it seems plausible to say that $X$ is the aim of science just in case science makes progress when we increase, accumulate, or get closer to achieving $X$. Discussions of the aim of science were brought to the forefront with van Fraassen's The Scientific Image, in which he characterized scientific realism in part as the view that science aims to give us true theories. His own preferred anti-realist view, constructive empiricism, holds that the aim of science is to give us empirically adequate theories, i.e. theories that are correct about the observable aspects of the world. Interestingly, this points to the possibility of an empiricist account of scientific progress according to which science makes progress precisely when our theories become increasingly empirically adequate. Such a view has not yet been explicitly defended in the literature.

Relatedly, there is an underexplored connection between scientific progress and more traditional versions of scientific realism. Scientific realism is usually characterized as the idea that currently accepted theories are probably and/or approximately true, but there are wellknown difficulties with realism thus defined - e.g., the fact that most scientific theories that have ever been accepted have later been discarded (Laudan 1981a). Van Fraassen's suggestion to define realism purely in terms of the aim of science has seemed too weak to capture the essence of the debate (Chakravartty 2017), since it makes no requirement that the aim is achieved to any extent. Some, e.g. Bird (2007) and Niiniluoto (2017), have suggested that the most plausible form of scientific realism can be defined in terms of whether science tends to make genuine progress with regard to the unobservable aspects of the world. Of course, whether this constitutes a defensible form of scientific realism ultimately depends on what scientific progress consist in.

\footnotetext{
${ }^{11}$ Rowbottom (2015) criticizes the truthlikeness account for not being able to accommodate scientific progress of this kind.
} 


\section{Further reading}

Alternative overviews of philosophical issues concerning scientific progress are provided by Niiniluoto (2015) and Bird (2015). Niiniluoto (2015) provides an accessible summary of the truthlikeness account (but see Niinliluoto 1980 for a fuller treatment). The problem-solving account is nicely summarized by Laudan (1981b; see Laudan 1977 for the full treatment). Bird (2007) prompted a resurgence in the debate about scientific progress with his knowledgebased epistemic account. Dellsén (2016) uses Bird's account as a foil to develop his understanding-based noetic account. 


\section{References}

Bangu, S. 2015. Progress, Understanding, and Unification. In I. D. Toader, G. Sandu \& I. Pârvu (eds.), Romanian Studies in Philosophy of Science. Cham: Springer, pp. 239-253.

Barnes, E. 1991. Beyond verisimilitude: A linguistically invariant basis for scientific progress. Synthese 88: 309-339.

Bird, A. 2007. What is scientific progress? Noûs 41: 64-89.

Bird, A. 2008. Scientific progress as accumulation of knowledge: A reply to Rowbottom. Studies in History and Philosophy of Science 39: 279-281.

Bird, A. 2015. Scientific Progress. In P. Humphreys (ed.), The Oxford Handbook of Philosophy of Science. Oxford: Oxford University Press, pp. 544-563.

Chakravartty, A. 2017. Scientific Realism. In E. N. Zalta (ed.), The Stanford Encyclopedia of Philosophy (Summer 2017 Edition), URL <https://plato.stanford.edu/archives/sum2017/entries/scientific-realism/>.

Cohen, L.J. 1980. What has Science to do with Truth? Synthese 45: 489-510.

Dellsén, F. 2016. Scientific Progress: Knowledge versus Understanding. Studies in History and Philosophy of Science 56: 72-83.

Dellsén, F. 2017. Understanding without Justification or Belief. Ratio 30: 239-254.

Dellsén, F. 2018. Scientific Progress, Understanding, and Knowledge: Reply to Park. Forthcoming in Journal for General Philosophy of Science.

Glanzberg, M. 2016. Truth. In E. N. Zalta (ed.), The Stanford Encyclopedia of Philosophy (Winter 2016 Edition), URL = <https://plato.stanford.edu/archives/win2016/entries/truth/>.

Grimm, S. 2006. Is Understanding a Species of Knowledge? British Journal for the Philosophy of Science 57: 515-535.

Hills, A. 2016. Understanding Why. Noûs 49: 661-688.

Kuhn, T. S. 1970. The Structure of Scientific Revolutions (2nd ed.). Chicago, IL: University of Chicago Press.

Kvanvig, J. (2009). "The Value of Understanding", in A. Haddock, A. Millar, and D. Pritchard (eds.), Epistemic Value. New York: Oxford University Press, pp. 95-111.

Laudan, L. 1977. Progress and its Problems: Toward a Theory of Scientific Growth. Berkeley: University of California Press.

Laudan, L. 1981a. A confutation of convergent realism. Philosophy of Science 48: 19-49.

Laudan, L. 1981b. A problem-solving approach to scientific progress. In I. Hacking (ed.), Scientific Revolutions. Oxford: Oxford University Press, pp. 144-155. 
Miller, D. 1974. Popper's qualitative theory of verisimilitude. British Journal for the Philosophy of Science 25: 166-177.

Mizrahi, M. 2013. What is scientific progress? Lessons from scientific practice. Journal for General Philosophy of Science 44: 375-390.

Niiniluoto, I. 1977. On the truthlikeness of generalizations. In R. E. Butts and J. Hintikka (eds.), Basic Problems in Methodology and Linguistics. Dordrecht: Reidel, pp. 121-147.

Niiniluoto, I. 1980. Scientific progress. Synthese 45: 427-462.

Niiniluoto, I. 1984. Is Science Progressive? Dordrecht: Reidel.

Niiniluoto, I. 1987. Truthlikeness. Dordrecht: Reidel.

Niiniluoto, I. 2014. Scientific progress as increasing verisimilitude. Studies in History and Philosophy of Science 46: 73-77.

Niiniluoto, I. 2015. Scientific progress. In E. N. Zalta (Ed.), The Stanford Encyclopedia of $\begin{array}{lllll}\text { Philosophy } & \text { (Summer } & 2015 & \text { ed.). }\end{array}$ <https://plato.stanford.edu/archives/sum2015/entries/scientific-progress/>.

Niiniluoto, I. 2017. Optimistic realism about scientific progress. Synthese 194: 3291-3309.

Oddie, G. 1986. Likeness to Truth. Dordrecht: Reidel.

Park, S. 2017. Does Scientific Progress Consist in Increasing Knowledge or Understanding? Journal for General Philosophy of Science, DOI: 10.1007/s10838-017-9363-2.

Popper, K.R. 1972. Objective Knowledge. Oxford: Clarendon Press.

Rowbottom, D.P. 2008. N-rays and the semantic view of scientific progress. Studies in History and Philosophy of Science 39: 277-278.

Rowbottom, D.P. 2010. What scientific progress is not: Against Bird's epistemic view. International Studies in the Philosophy of Science 24: 241-255.

Rowbottom, D.P. 2015. Scientific progress without increasing verisimilitude: In response to Niiniluoto. Studies in History and Philosophy of Science 51: 100-104.

Saatsi, J. 2016. What is theoretical progress of science? Synthese, DOI: 10.1007/s11229-0161118-9.

Sliwa, P. 2015. Understanding and Knowing. Proceedings of the Aristotelian Society 115: 5774.

Tichý, P. 1974. On Popper's definitions of verisimilitude. British Journal for the Philosophy of Science 25: 155-160.

van Fraassen, B.C. 1980. The Scientific Image. Oxford: Oxford University Press. 
Wilkenfeld, D.A. 2017. "Understanding without Believing", in S. Grimm, C. Baumberger, and S. Ammon (eds.), Explaining Understanding: Perspectives from Epistemology and Philosophy of Science. New York, NY: Routledge, pp. 318-334. 